\title{
Zur Abgrenzung und Bestimmung von Planungsregionen
}

Günther Schumacher

\section{Begriff und Aufgabe der Region}

Der Begriff «Region» bedeutete früher eine bestimmte Landschaft, welche sich als besondere territoriale Einheit hervortat. Heute wird aber dieser Terminus auf vielfältigste Art angewandt, so daß sich keine eindeutige Definition mehr geben läßt. Meist versteht man jedoch unter Region einen zweckbestimmten Raum (Verwaltungsgebiet, Agglomeration, Städtegruppe). Ablehnen muß man aber jene Arten von Regionalbezeichnungen, die nur dann angewendet werden, wenn in einem Raum neue Probleme, z. B. Verkehr, Wirtschaft, entstanden sind.

Man kann sagen, daß eine Region weder ein selbstdefiniertes noch ein von Natur gegebenes Objekt ist. Sie ist ein Begriff, eine Einheit des Denkprozesses und ist mit den Merkmalen, welche für ein Gebiet oder ein Flächenproblem relevant sind, beschreibbar.

Es stellt sich die Frage, welchen Platz und welche Bedeutung die Region als Raumeinheit im Rahmen der Landesplanung einnimmt.

Der Landesplaner braucht die Region als Arbeitsinstrument, um durch sie seine Kenntnisse und $\mathrm{Er}$ kenntnisse der territorialen und der gesellschaftlichen Einheit zu umschreiben. In diesem Sinne ist die Region eine wichtige Basis aller landesplanerischen Initiativen. Der Landesplaner versteht unter Region ein Gebiet, in dem sich die Natur- und Kulturelemente so verflechten, daß sie irgendwie eine individuelle und hinsichtlich der verschiedenen natürlichen, wirtschaftlichen und sozialen Eigenschaften eine organisch-räumliche Einheit, die sich von den anderen Einheiten unterscheidet, gestalten. Jede Region grenzt wiederum an eine andere benachbarte Region. Verschiedene benachbarte Regionen zusammen bilden wiederum eine übergeordnete größere Regionseinheit.

Die Landesplanung hat die Regionen gemäß der kulturgeographischen Gegebenheiten in der Landschaft zu koordinieren. In diesem Sinne sagte Lewis Mumford: "The re-animation and re-building of regions as deliberate work of collective art, is the grand task of politics for comming generation.» Die Region ist somit auch Forschungsobjekt: sie soll die vergangenen und gegenwärtigen Strukturen der Landschaft und der menschlichen Gesellschaft interpretieren und nach Möglichkeiten einer Verbes- serung und Umgestaltung in Übereinstimmung mit den zu erwartenden Entwicklungen suchen. Im gegenwärtigen Zeitpunkt ist es aber leider immer noch so, daß die Landesplaner ziemlich weit davon entfernt sind, einheitliche Grundsätze und Konzepte für eine Regionalisierung vorzulegen bzw. zu praktizieren.

\section{F. Lodewig schrieb bereits im Jahre 1941 folgendes:}

"Die Regionalplanung bezweckt, den Boden eines ganzen Wirtschaftsbeckens (Region) seiner jeweils besten Nutzung (Wohnung, Industrie, Landwirtschaft, Erholungsgebiete usw.) zuzuführen. Für die Wohnstätten sollen dabei gleichzeitig gesundheitlich und verkehrstechnisch günstigste Bedingungen geschaffen werden.»

Ein anderer bekannter Landesplaner, J. Umlauf, erläuterte das Problem der Regionalisierung wie folgt: "Die erste Voraussetzung für einen guten Nutzeffekt der regionalen Planung ist die richtige Abgrenzung des Planungsgebietes. Echte, lebendige Selbstverwaltung ist nur möglich, wenn wirklich dringende, aktuelle Bedürfnisse sowie starke nachbarschaftliche, gemeinsame Interessen vorliegen. Die Abgrenzung solcher ,eigenständiger' Planungsgebiete ist bereits ein Bestandteil der Landesplanungsarbeit; ihr Erfolg hängt wesentlich davon ab.»

\section{Regionalisierung und Regionstypen}

In der Planungsliteratur findet man eine große Anzahl von divergierenden Auffassungen über die Region und ihre Begrenzung, während über die Funktion der Region an sich im großen und ganzen übereinstimmende Auffassungen zu erkennen sind. Trotz vieler Vorschläge dürfte auch in Zukunft eine «Patent-Planungsregion» nicht realisierbar sein. Die Planungsregion heutiger Prägung sollte dem allgemeinen Bedürfnis entsprechen und aufgrund verschiedener Regionalisierungsmethoden gestaltet werden. Nach Prüfung der verschiedenen Methoden sollte gemäß der bestehenden Bedürfnisse und Ziele der Entscheid gefällt werden. Wir zählen hier verschiedene Regionstypen auf, um dem Leser ein Bild der möglichen Varianten zu geben: 


\section{Regionen}

- natürliche

- historische

- sprachliche

- administrative

- statistische

- ökonomische

- verkehrstechnische

- versorgungstechnische

Aus der Kombination verschiedener dieser Partialregionen entsteht dann die funktionale Planungsregion (französisch «région de programme»). $\mathrm{Ob}$ nun aber diese Planungsregion mehr in Richtung eines homogenen oder eines heterogenen Raumes zu liegen kommt, hängt wohl von Fall zu Fall von dem zu untersuchenden Landesteil ab.

Funktionale Planungsregionen kann man nach O. Boustedt und H. Ranz wie folgt umschreiben: «Bei funktionalen Räumen kommt es darauf an, die Gebiete zusammenzufassen, in denen die Einzelerscheinungen verschiedenster Art im gemeinsamen Ordnungsprinzip der gegenseitigen Lagebeziehungen zu einer Einheit zusammengeschlossen sind. Das wesentlichste Merkmal ist somit die Abgrenzung von Räumen aufgrund der Richtung ihrer allgemeinen Verkehrsorientierung; eine funktionale Raumeinheit stellt stets einen Raum dar, der in seiner Ausdehnung und Begrenzung durch die Anziehungskraft bzw. Reichweite eines übergeordneten sozio-ökonomischen Schwerpunktes bestimmt wird. Das klassische Beispiel für eine derartige Raumgliederung ist die Gebietseinteilung nach den Einzugsbereichen zentraler Orte. Hier wird nicht mehr nach Merkmalsgleichheit oder -verwandtschaft der Teilgebiete gefragt, sondern lediglich nach der räumlichen Zugehörigkeit einzelner Teilgebiet zu übergeordneten Zentren. In struktureller Hinsicht können und werden die Teilgebiete vielfach heterogen sein, ja sie müssen es sogar aus de: Natur der Sache her sein, denn die funktionalen Zusammenhänge ergeben sich ja gerade aus dem Austauschbedürfnis unterschiedlich strukturierter Räume.»

Mancherorts sind aber die Funktionen (z. B. in der Wirtschaft) so verflochten, daß es schwierig ist, $\mathrm{zu}$ trennen und zu kombinieren. Die Schwierigkeiten nehmen vor allem dann zu, wenn für die zu bestimmende Region nicht nur eine, sondern zwei oder mehrere Orte mit höheren funktionalen Komponenten zu analysieren sind.

Die aus einem funktionalen Bedürfnis gestaltete Region besitzt keine absoluten Konstanten. Jede Verlagerung innerhalb der funktionalen Region verursacht Schwerpunkt- und Typenänderungen. Aus diesem Grunde kann jeder Versuch einer "ewig" festgelegten Regionalisierung als verfehlt und reali- tätsentfremdend bezeichnet werden. Zudem ist auch bis heute kein unveränderliches und allgemeingültiges Regionalisierungsschema bekannt. Das Suchen nach einer funktionalen Region mit möglichst vielen optimalen Eigenschaften ist ein ununterbrochener Prozeß und nie das Ergebnis einer einmaligen Untersuchung.

\section{Landwirtschaft, Industrie und Dienstleistungen in der Region}

Es ist bekannt, daß für die Bestimmung einer zentralörtlichen Gegebenheit die Dienstleistungen die größte Bedeutung haben. Landwirtschaft und Industrie erhalten Impulse durch Aufwertungen zentralörtlicher Dienstleistungen.

Die Bedeutung von Landwirtschaft und Industrie ist für die Regionalisierung von zweitrangigem Einfluß. J. Grendelmeier hält dies wie folgt fest: «Im Gegensatz zu den zentralen Dienstleistungseinrichtungen findet bei industriellen Einrichtungen kein direkter Kontakt von Produzent und Konsument statt. Zudem werden infolge zunehmender Spezialisierung der Betriebe und immer größerer Vielfältigkeit der Produkte immer weiter verstreute Verteilerzentren von Industriebetrieben aus beliefert. Die Transportkosten spielen eine abnehmende Rolle. Absatzgebiete der Industrien und Absatzgebiete der Zentren decken sich immer weniger. Dasselbe kann im abgeschwächten Maße von der Landwirtschaft gesagt werden. Die Versorgung der Verteilerzentren mit industriellen Erzeugnissen ist für die Abgrenzung einer Region daher nicht, mit landwirtschaftlichen Produkten nur zum Teil relevant.

Aus diesen Gründen können Industrie und Landwirtschaft dispers verteilt sein. Die Landwirtschaft ist es aus Naturgegebenheit ohnehin. Industriestandorte sind aus dieser Sicht gesehen somit für die Regionalbildung nicht relevant. Allerdings bestehen gerade zwischen Industrie und Dienstleistungen aus Gründen des Arbeitsmarktes und der von der Industrie benötigten Dienstleistungen enge Beziehungen, weshalb Industriebetriebe die Nähe von Zentren benötigen.»

\section{Probleme der Regionalbildung}

Je nach dem angestrebten Ziel kann man Regionen nach einzelnen Charakteristiken oder aus Kombinationen verschiedener Eigenheiten bestimmen. Dies ist entweder auf partiale oder auf synthetische Art und Weise möglich. Die partiale Art beurteilt ein Gebiet nach einem einzigen Kriterium; die synthetische hingegen verwendet Merkmalskombina- 


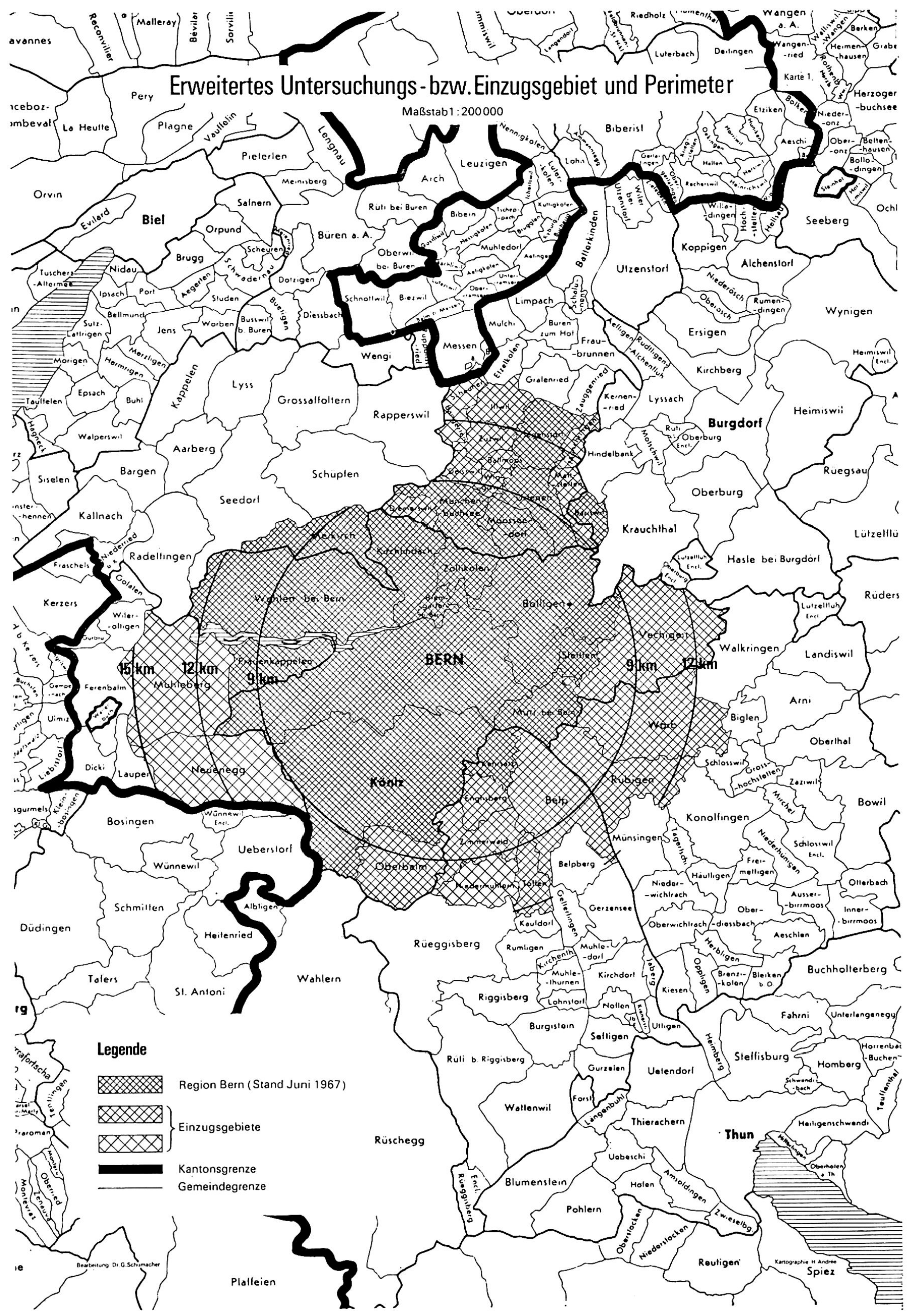


tionen oder eine Überlagerung mehrerer partialer Kriterien, woraus sich die funktionale Region ergibt. Folgende Kriterien lassen sich anführen:

Topographie: Höhenzüge, Wasserscheiden, Flußtäler

Wälder: große Waldflächen

Kulturelle Beziehungen zum Zentrum: vor allem Theater, Veranstaltungen, Mittel- und höhere Schulen

Wirtschaftliche Beziehungen zum Zentrum: Dienstleistungsbetriebe, Industrie

Technische Beziehungen zum Zentrum oder topographisch bedingt: Energieversorgung, Wasser, Abwasser, Kehricht

Verkehrseinrichtungen und -verbindungen: öffentliche Verkehrsmittel, Straßen

Durch eine Kombination dieser Merkmale kann versucht werden, den Grad der Zugehörigkeit einer Gemeinde zu einer Region festzustellen. Daneben können noch weitere Gründe für die Einbeziehung in die Region sprechen, beispielsweise die Auffüllung eines Regionsgebietes oder mehrerer Regionsgebiete.

Im Bergland kann es vorkommen, daß eine Region "partial» durch die Topographie als fest umrissenes Gebiet gegeben ist. Im Mittelland dagegen wird wahrscheinlich durchwegs eine funktionale Regionseinheit mit Hilfe einer Merkmalskombination gesucht werden müssen. Vor rund 25 Jahren hat H. Gutersohn zur Frage der Planungsregion bereits Stellung bezogen. Die Problematik hielt er wie folgt fest: "Schwierig wird die Gebietstrennung da, wo keine natürlichen Grenzen zu scharfer Scheidung führen. Dies ist namentlich im Schweizer Mittelland der Fall, und hier vor allem in Gegenden, die über flache Rücken oder weite Talungen miteinander in Beziehung stehen. Kaum behindert, flutet vom Haupttal die eine Kulturlandschaft bestimmter Prägung in die breiten Nebentäler hinein, mit ihren Siedlungs- und Wirtschaftsformen jegliche Grenze verwischend. Grenzziehung tut hier in allen Fällen der Wirklichkeit und ihren inneren Zusammenhängen Gewalt an und kann deshalb nie völlig befriedigen; und doch ist auch hier eine solche Grenzziehung anzustreben, soll die planerische Arbeit eine Basis erhalten. In diesen schwierigen Fällen sind in erster Linie die Kernräume festzustellen, in denen die vorwiegenden Eigenschaften am klarsten zur Geltung gelangen. Um diese Grenzräume legt sich aber keine Grenzlinie, sondern ein Grenzsaum, dessen Elemente teils mehr mit der einen, teils mehr mit der anderen Seite verbunden sind. Die Grenzsäume sind ,Kampfsäume'. Je verwickelter die Verhältnisse, um so notwendiger ist die Untersuchung der Grundlagen, um so größer sind auch die Probleme, die hier der Regionalplanung harren. Diese Grenz- säume sind auch in erster Linie jene Notstandsgebiete, die gestaltende Maßnahmen erheischen.» Eine funktionale Region wird in erster Linie durch die Verkehrs- und Wirtschaftsattraktivität des Zentrums bestimmt.

Für die Verkehrsregion konnten anhand der Region Bern bereits empirisch einige Meßdaten erarbeitet werden. (Karte 1; beachte: die Region Bern wird im Norden durch die Region Aarberg_Lyß und im Nordosten durch die Region Burgdorf begrenzt.) Zunächst ist festzustellen, wo die gegebenen Grenzen eines «regionsreifen» Zentrums liegen. Von diesem aus wählt man dann einen inneren, mittleren und maximalen Perimeter, durch die verschiedene Gemeinden angeschnitten bzw. ganz einbezogen werden. Es ist darauf zu achten, daß der maximale Perimeter einer schweizerischen Planungsregion im Prinzip nicht größer als $14-16 \mathrm{~km}$ (äußerer Perimeter) sein sollte (außer, wenn mit einem Verkehrsmittel ein verhältnismäßig geringerer zeitlicher Aufwand notwendig ist, ins Zentrum zu gelangen). Für die Abgrenzung einer Region kamen wir zu folgenden empirischen Anhaltspunkten:

Innerer Perimeter: Dieser wird von mindestens durch zwei von außen gegebenen «Fixpunkten» festgelegt; Fixpunkte sind in diesem Rahmen die innersten Begrenzungspunkte der zu begrenzenden Region (z. B. Topographie, Wasserscheide, direkter Einzugsbereich der nächstliegenden regionsreifen Zentren). Die innerhalb dieses inneren Perimeters gelegenen Gemeinden gehören zur Region und stehen mit dem Zentrum in engem Zusammenhang (= direkte Zuordnung). Der innere Perimeter der Region Bern liegt beispielsweise bei $9 \mathrm{~km}$.

Mittlerer Perimeter: Dieser liegt etwa im Mittel zwischen innerem und äußerem Perimeter (mitticre $\mathrm{Zu}$ ordnung). Der mittlere Perimeter erfaßt in der Regel jene Gemeinden, die bereits durch den inneren Perimeter angeschnitten wurden. Der Einzugsbereich der nächstliegenden Regionen wie auch z. B. topographische Grenzen können hier schon stark abgrenzend wirken.

Äußeren Perimeter: Dieser erfaßt jene Gemeinden, die an Verkehrsachsen liegen, die auf das Regionszentrum zulaufen. Weiterhin umschreibt er den Kreis der sogenannten Auffüllgemeinden. Der äußere Perimeter charakterisiert sich somit durch eine schwache bzw. auffüllende Zuordnung.

\section{Weg- und zeitbestimmte Kriterien}

Weg- und zeitbestimmte Kriterien sind sowohl für den Gesamtdurchschnitt wie für den Einzelfall zur Zurücklegung des Arbeitsweges zu untersuchen und $\mathrm{zu}$ bestimmen. Für die größten Regionen der Schweiz (z. B. Zürich) dürften die mittleren Fahr- 


\section{Mittlere Fahrzeiten und Entfernungen öffentlicher Verkehrsmittel}

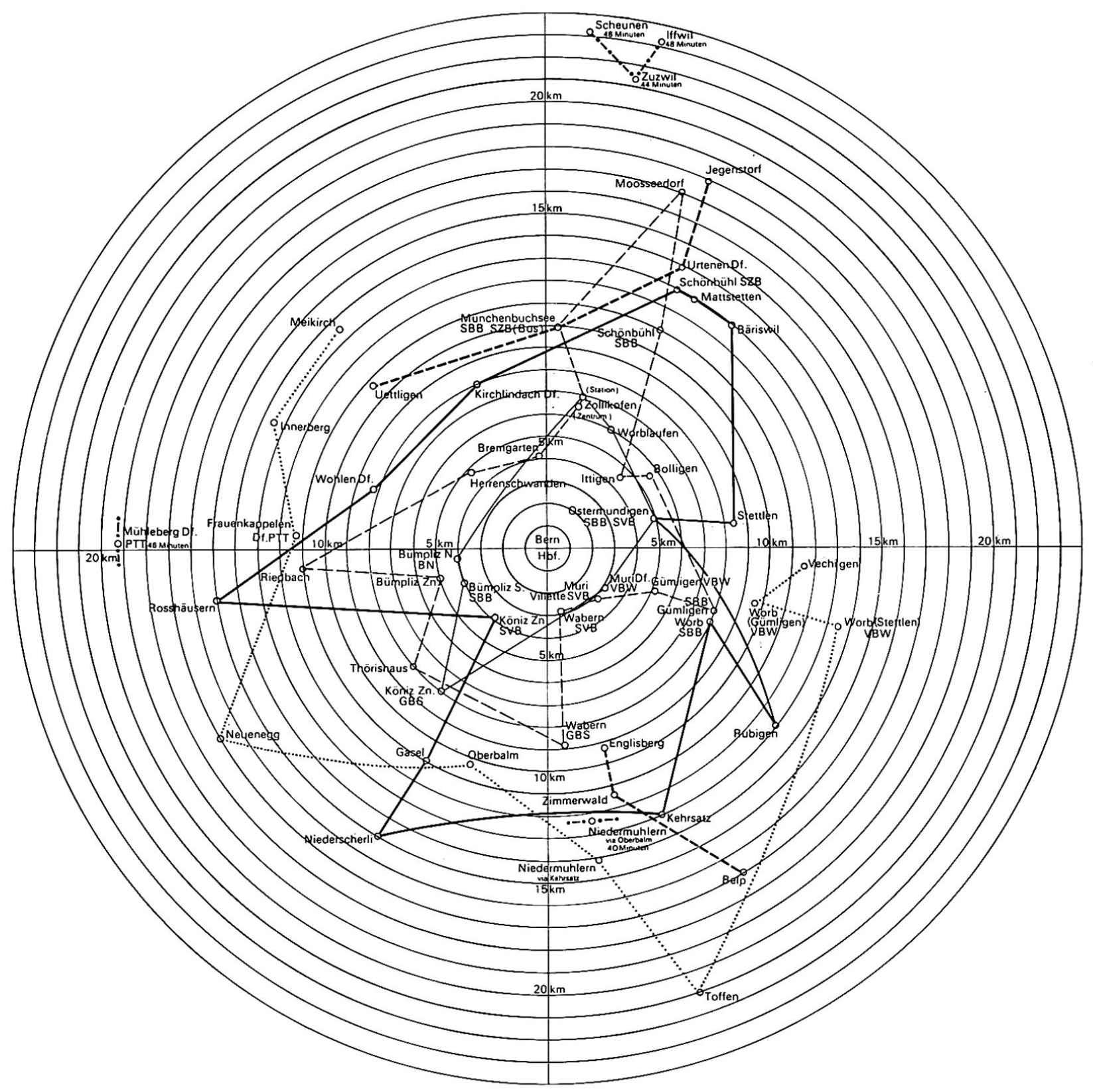

Fahrzeiten

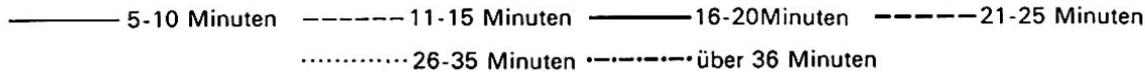


zeiten mit dem öffentlichen Verkehrsmittel bei 25-35 Minuten und mit dem privaten Motorfahrzeug bei 20-25 Minuten liegen. Für weniger große Regionszentren (z. B. Bern) dürften die mittleren Fahrzeiten bei Benützung der öffentlichen Verkehrsmittel im Mittel bei 20-25 Minuten und maximal bei 30 Minuten liegen; für die privaten Verkehrsmittel liegen die Fahrzeiten im Mittel bei 17 Minuten und maximal bei 20-22 Minuten.

Wie kommt man zu diesen Angaben? Nach der Festlegung des inneren, mittleren und äußeren Perimeters einer Region läßt sich folgendes Vorgehen aufzeichnen, um die approximativen zeitlichen Grenzen zu erhalten:

Öffentliches Verkehrsmittel

Mittlere Fahrzeit: Man multipliziere den mittleren Perimeter (Kilometerangabe) mit 2 und erhält somit die mittlere Fahrzeit in Minuten.

Maximale Fahrzeit: Man multipliziere den äußeren Perimeter (Kilometerangabe) mit 2 und erhält die maximale Fahrzeit in Minuten.

Private Verkehrsmittel

Mittlere Fahrzeit: Man multipliziere den inneren Perimeter (Kilometerangabe) mit 2 und erhält somit die mittlere Fahrzeit in Minuten.

Maximale Fahrzeit: Man multipliziere den mittleren Perimeter (Kilometerangabe) mit 2 und erhält somit die maximale Fahrzeit in Minuten.

Beispiel:

Voraussetzung: Perimeterbestimmung einer Region
a) innerer Perimeter $7 \mathrm{~km}$
b) mittlerer Perimeter $10 \mathrm{~km}$
c) äußerer Perimeter $13 \mathrm{~km}$

Mittlere Fahrzeiten (Minuten)

Öffentliche Verkersmittel $10 \times 2=20$ (18-22)

Private Verkehrsmittel: 7 × $2=14$ (12-16)

Maximale Fahrzeiten (Minuten)

Öffentliche Verkehrsmittel $13 \times 2=26$ (24-28)

Private Verkehrsmittel $10 \times 2=20$ (18-22)

In Klammern finden sich Angaben über den mittleren Streuungsbereich der erhaltenen Werte. Für jene Regionen deren äußerer Perimeter auf $14 \mathrm{~km}$ und darüber zu liegen kommt, ist der mittlere Streuungsbereich mit +5 und -5 vom erhaltenen Wert einzusetzen. Úberschreitet der äußere Perimeter nicht $13 \mathrm{~km}$, so genügt ein Streuungsbereich von +2 und -2 .

Möchte man beispielsweise für den Kanton Bern die Anzahl der zu schaffenden (funktionalen) Regionen schätzen, so dürfte dies ungefähr folgendes Ergebnis zeigen. Wir schätzen, daß im Durchschnitt der äußere Perimeter aller Regionen zwischen 9,5 km und $11 \mathrm{~km}$ liegen wird. Der Kanton Bern hat eine Fläche von $6887 \mathrm{~km}^{2}$; die mittlere Regionsfläche würde somit bei $9,5 \times 9,5 \times 3,14$ bis $11 \times 11 \times 3,14$ Quadratkilometer liegen. Dies ergibt für den Kanton Bern schätzungsweise 18-24 Planungsregionen.

\section{Beispiel der nach weg- und zeitbestimmten Kriterien untersuchten Region Bern}

Für die in den möglichen Einzugsbereich fallenden Gemeinden bzw. Ortschaften wurden für die öffentlichen Verkehrsmittel die in den Fahrplänen angegebenen Reisezeiten (gemittelte Werte) errechnet; Die Distanzen sind ebenso dem amtlichen Kursbuch entnommen. Die Fahrzeiten (einschl. Distanzen) für den privaten Motorfahrzeugverkehr wurden während der Tageszeit bei normalen Verkehrsverhältnissen und Geschwindigkeiten gemessen. Die beiden Karten 2 und 3 stellen das Ergebnis dieser Untersuchungen dar.

Die Karte 2 zeigt ein ziemlich unregelmäßiges Bild der Verkehrsverhältnisse auf. Die Fahrzeiten variieren je nach Verkehrsmittel (Städtische Verkehrsbetriebe, Vorortsbahnen, SBB usw.). Bei genauem Betrachten lassen sich aber auch für jene Leser, die die Region Bern weniger gut kennen, die wichtigsten Verkehrsachsen herauslesen. Interessant ist auch das Herausfallen der Gemeinden Iffwil, Scheunen und Zuzwil aus dem gesamten Bild; es handelt sich um typische Auffüllgemeinden von an und für sich schwacher Zuordnung. In bezug auf die Auffüllgemeinden ist die Kenntnis der Gemeindetypen, auf die hier nicht näher eingegangen werden kann, sehr von Nutzen.

Die Karte 3 bietet ein viel ruhigeres und ausgeglicheneres Bild. Die Fahrzeiten nehmen vom Kern (Bern $\mathrm{HB}$ ) ausgehend verhältnismäßig regelmäßig zu. Die ausgesprochenen Verkehrsachsen haben eine weit weniger große Bedeutung als bei den öffentlichen Verkehrsmitteln. Interessant ist auch wie die Auffüllgemeinden (Iffwil, Scheunen, Zuzwil) sich hier viel näher und besser an den übrigen Regionskreis anschmiegen.

Für die Leser sei noch angeführt, daß es interessant ist, diese beiden Diskussionskarten auf einen durchsichtigen Film aufzutragen, um sich damit einen direkten Vergleich für jede Gemeinde bzw. Ortschaft in bezug auf die Verkehrsgunst machen zu können.

\section{Weitere Abgrenzungskriterien}

Im allgemeinen soll man nur dort die Kantonsgrenze überschreiten, wo es unbedingt erforderlich ist. Diese Aussage ist nicht als Unterstützung für den Kantönligeist gedacht, sondern ergibt sich aus den meist sehr verschiedenen Baugesetzen, wodurch sich zum Teil erhebliche Schwierigkeiten ergeben können.

$\mathrm{Zu}$ berücksichtigen sind ferner auch ältere Gemeindeverbände (z. B. Fürsorge- und Schulverbände), zentralisierte Post, Kulturverbände (z. B. Theaterund Konzertgemeinschaften) und Verwaltungsabkommen (Zuständigkeit einer Gemeindeschrei- 


\section{Mittlere Fahrzeiten und Entfernungen für die privaten Motorfahrzeuge}

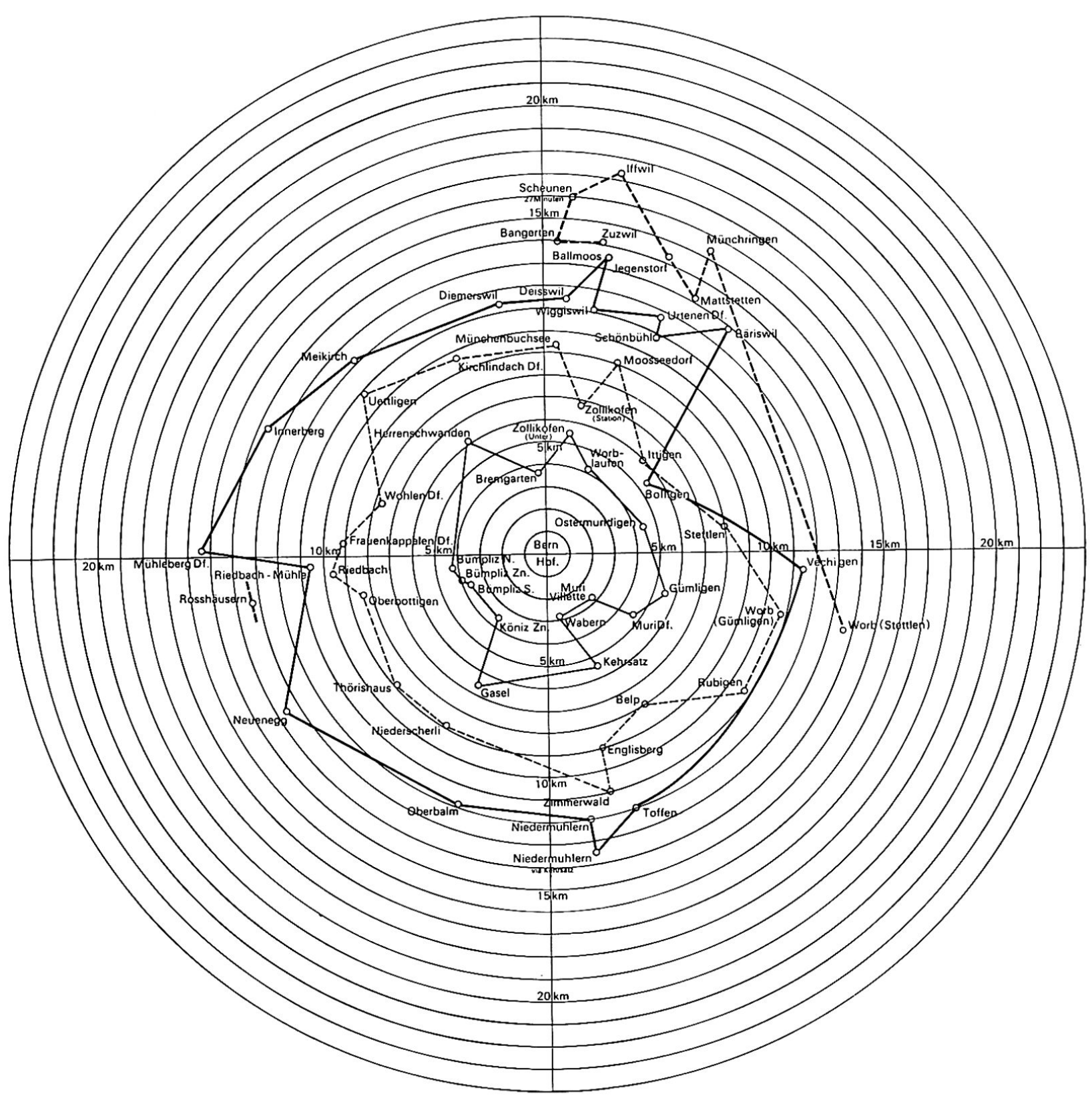

Fahrzeiten 
berei für mehrere Gemeinden). Diese Verbände sind wegen ihrer stillen Tätigkeit und ihrer langen Bewährung selten direkt erkennbar, doch sind sie meist tief im Volksbewußtsein verwurzelt. Hier gilt es eine gute Information und Kenntnis des zu regionalisierenden Gebietes zu besitzen, um nicht wegen ein paar weniger wichtigeren Abgrenzungskriterien gewachsene Einheiten zerstören zu wollen.

\section{Von der Regionalabgrenzung zur Regional- organisation und Regionalfinanzierung}

Ein erster Schritt zur Regionalorganisation war bis heute mehr oder weniger die Vereinsform auf privatrechtlicher Grundlage. Diese genügt aber bei weitem vielerorts nicht mehr, weil die Durchführung der Regionalpläne nicht gesichert werden konnte. Auch ergibt sich oft die paradoxe Situation, daß die Stadtplanung mit allen Vollmachten der öffentlichen Hand gesegnet ist, während die Regionalplanung mancherorts kompetenzarm ist oder um ihre nackte Existenz kämpfen muß. Ein erster Schritt zur Beseitigung dieser «Kinderkrankheiten» ist, die Schaffung sogenannter Gemeindeföderationen oder Gemeindeverbände in Erwägung zu ziehen. In den Gemeindeföderationen erhält jeder Bürger Mitspracherecht und wird nicht wie in den meisten Regionalvereinen durch eine kleine Gruppe von "Besserwissenden» bevormundet. Die Gemeindeföderation wäre ähnlich wie der Aufbau unseres Bundesstaates zu statutieren. Mitglieder sind demnach nicht nur die Gemeinden, sondern auch jeder Bürger, der damit ein direktes Verhältnis zur Region und ihrer Politik erhält. Die Föderationsgemeinden verpflichten sich, gewisse Aufgaben an das übergeordnete Organ abzutreten. Die Aufgaben der Föderation sind entweder durch spezielle Steuern oder durch Belastung der Gemeinden finanziell zu sichern.

Dort, wo die Gemeindeföderation nicht mehr genügt, kann die straffere Form des Regionalverbandes gewählt werden. Voraussetzung dafür ist die Schaffung und Anerkennung einer regionalen Autonomie. Die Autonomie des Regionalverbandes ist ähnlich jener der Gemeinden ( $=$ administrativ, vermögensrechtlich, statutarisch), d. h. daß die Bürger der Region berechtigt werden, ihre regionalen Aufgaben im Rahmen von Verfassung und Gesetz selbständig zu lösen. Dies kann mit Hilfe von Abstimmungen, eines großen Regionalrates, Fachkommissionen und Wahlbehörden in die Tat umgesetzt werden.

Wie wir funktional bestimmte Regionen und ihre rechtliche und wirtschaftliche Organiasation miteinander abstimmen, in Einklang bringen und flexibel genug gestalten können, wird uns die nächste
Zukunft lehren. Heute sieht es auf jeden Fall so aus, daß eine Lösung erst durch gesammelte Erfahrung und nicht durch ein im voraus festgelegtes Schema gesucht werden muß.

\section{Literatur}

Boustedt O., Ranz H.: Regionale Struktur- und Wirtschaftsforschung. Bremen 1957.

Deutsche Akademie für Städtebau und Landesplanung: Dichteprobleme in Landesplanung und Städtebau. Sonderausgabe 1967, Düsseldorf.

Grendelmeier J.: UUber die Planung optimaler Planungsregionen. ORL-Institut, Arbeitsgruppe Planungsrichtlinien. Zürich 1965.

Gutersohn H.: Zur Frage von Planungsregionen. Separatdruck aus: «Plan», I. Jg., Mai 1944.

Lenort N. J.: Entwicklungsplanung in Stadtregionen. Köln und Opladen 1961.

Lodewig F.: Regionalplanung. In: Schweizerische Technische Zeitschrift, Jg. 16, Juli 1941.

Müller A.H.: Rechtsträger für regionale Aufgaben. Diss. Basel 1967.

Mumford L.: The Culture of Cities. London 1938.

Schumacher G.: Zur Abgrenzung und Größe der Region Bern. In: Berner Beiträge zur Stadt- und Regionalforschung. Jg. 1967, Heft 1, Bern.

Stocker P., Risch P.: Einkommenslage und Wirtschaftsstruktur des Kantons Bern. Wachstumspolitische Lageanalyse und Skizze eines Förderungsprogramms. Berner Beiträge zur Nationalökonomie. Bd. 7, Bern 1968.

Stutz W.: Die kommunalen Zweckverbände im Kanton Aargau. Diss. Zürich 1964.

Umlauf J.: Regionalplanung im Ruhrgebiet. Plan $17,1960$.

Veröffentlichung der Akademie für Raumforschung und Landesplanung: Zur Methodik der Regionalplanung. Bd. 41, Hannover 1968.

Vriser I.: Die Region in der Landesplanung. ORL-Institut. Zürich 1965.

Werczberger E.: Untersuchung über die Pendlerregionen und Einteilung der Schweiz in Arbeitsmarktregionen. ORL-Institut, Zürich 1965.

Winkler E.: Regionalbeschreibung als Grundlage geographischer Landesaufnahme und Landesplanung. In: Geographica Helvetica 8/1953.

Winkler E.: Theoretische Aufgaben der Landesplanung. Nr. 35 der Arbeiten aus dem Geographischen Institut der ETH, Zürich 1960.

Winkler E., Schumacher G., Vriser I., Jacsman J.: Institutionen der Landes- und Regionalplanung in der Schweiz Ende 1965. ORL-Institut, Zürich 1965.

\section{Anmerkung}

1 Auf die Bedeutung der Kursdichten kann in diesem Zusammenhang nicht eingegangen werden. 